\title{
EVN and MERLIN observations of III Zw 35
}

\section{A starburst continuum and an $\mathrm{OH}$ maser ring}

\author{
Y. M. Pihlström ${ }^{1}$, J. E. Conway $^{1}$, R. S. Booth ${ }^{1}$, P. J. Diamond ${ }^{2}$, and A. G. Polatidis ${ }^{1}$ \\ 1 Onsala Space Observatory, 43992 Onsala, Sweden \\ 2 Jodrell Bank Observatory, University of Manchester, Macclesfield, Cheshire, SK11 9DL, UK
}

Received 14 May 2001 / Accepted 31 July 2001

\begin{abstract}
EVN and MERLIN observations of the $18 \mathrm{~cm}$ continuum and $\mathrm{OH}$ maser emission in the luminous infrared galaxy III Zw 35 are presented. The continuum consists of two components. The first component is made up of a number of compact sources, resembling the types of objects observed in Arp 220 and Mrk 273. In III Zw 35 the origin of these compact components is unclear, and we discuss the possibility they are individual young radio supernovae. Alternatively they could be clusters or clumps of young supernova remnants. The second continuum component is diffuse and has an angular size of $\sim 460$ mas $(230 \mathrm{pc})$ and $T_{\mathrm{b}} \sim 10^{5} \mathrm{~K}$. We argue that this component is also powered by star formation. From the FIR flux we estimate a star formation rate of $19 M_{\odot} \mathrm{yr}^{-1}$, which in turn implies a supernova rate of $0.8 \mathrm{yr}^{-1}$, sufficient to power the diffuse radio emission. Turning to the $\mathrm{OH}$ maser emission we find, in agreement with earlier VLBI observations of III Zw 35, that the bright OH maser emission is concentrated in two compact clumps. However, in addition we observe diffuse maser emission which lies in a clear ring structure of radius $r \sim 22 \mathrm{pc}$. The two compact maser features occur at the tangent points of the ring. This geometry and their similar $1667 / 1665 \mathrm{MHz}$ line ratios suggests that the compact and diffuse masers appear different because of path length effects and not pumping or other physical conditions. A simple model with many clouds within a ring seems able to fit the main properties of both the diffuse and compact maser emission. We find a velocity gradient across the diffuse maser emitting region which confirms rotation. From this gradient we estimate a dynamical mass of $7 \times 10^{6} M_{\odot}$ within $r \sim 22$ pc. OH absorption is also present just outside the ring of maser emission. Both the $\mathrm{OH}$ maser emission and the continuum is weaker on the Eastern side of the source. We argue that this could be due to free-free absorption effects, which implies gas with an emission measure of $7 \times 10^{6} \mathrm{~cm}^{-6} \mathrm{pc}$.
\end{abstract}

Key words. galaxies: active - galaxies: individual: III Zw 35 - galaxies: ISM - masers - radio lines: galaxies radio continuum: galaxies

\section{Introduction}

Galaxies which emit most of their flux in the infrared are, depending on the strength of the infrared luminosities, further subdivided into luminous infrared galaxies (LIRGs, $L>10^{11} L_{\odot}$ ) and the ultra-luminous versions (ULIRGs, $\left.L>10^{12} L_{\odot}\right)$. The extreme infrared luminosities of these galaxies likely originate from dust grains heated by a central power source which may be either a nuclear starburst, an active galactic nuclei (AGN) or a combination of both. Mid-IR observations of a sample of ULIRGs are consistent with the majority (70-80\%) being starburst driven, while an AGN might dominate in $20-30 \%$ of the objects (Genzel et al. 1998). However, several of the ULIRGs probably contain both a starburst as well as an AGN. Veilleux et al. (1995) observed a larger sample of lower luminos-

Send offprint requests to: Y. Pihlström,

e-mail: ylva@oso.chalmers.se ity $\operatorname{IRAS}$ galaxies $\left(L>10^{10.5} L_{\odot}\right)$ and conclude that in these sources starbursts dominate, although they found a trend towards a higher incidence of AGN at higher IR luminosity. The most direct evidence so far for ULIRGs harbouring starbursts, are provided by VLBI observations of Arp 220 (Smith et al. 1998a) and Mrk 273 (Carilli \& Taylor 2000). These two sources both show compact unresolved components of a few pc in size with non-thermal brightness temperatures, consistent with supernovae or young supernova remnants.

In many cases $\mathrm{OH}$ megamasers are associated with LIRGs and ULIRGs. The standard model for those has involved a low gain molecular structure on scales 100 1000 pc amplifying background continuum. The necessary population inversion is best explained by pumping from IR photons. Such $\mathrm{OH}$ megamaser emission $\left(L_{\mathrm{OH}}=527 L_{\odot}\right)$ has been found in the LIRG III Zw 35 (Staveley-Smith et al. 1987) at a distance of $110 \mathrm{Mpc}$ 
(adopting $H_{0}=75 \mathrm{~km} \mathrm{~s}^{-1} \mathrm{Mpc}^{-1}$, which gives a linear scale of $\left.0.5 \mathrm{pc} \mathrm{mas}^{-1}\right)$. This galaxy is probably the result of a merger, displaying a double nucleus with a separation of about $9^{\prime \prime}$. While the Southern nucleus is weak in near-IR lines and lacks radio continuum emission, in the Northern nucleus near-IR, optical and radio continuum emission coincide (Chapman et al. 1990). This nucleus is also the site of the $\mathrm{OH}$ megamaser emission. VLA and MERLIN $18 \mathrm{~cm}$ continuum observations display an unresolved feature with a total flux density of around $40 \mathrm{mJy}$ (Chapman et al. 1990). More recent VLBA as well as global VLBI data (Trotter et al. 1997; Diamond et al. 1999) failed to show any continuum emission implying that it must be resolved out with these instruments.

In addition to resolving the continuum, the above mentioned VLBA and global VLBI observations also appear to resolve out a large part $(\sim 50 \%)$ of the $\mathrm{OH}$ maser emission, as compared to the single-dish spectra published by Staveley-Smith et al. (1987). The maser emission detected with these instruments is compact and mainly situated in two clumps (one of which has an interesting position - velocity diagram; Trotter et al. 1997; Diamond et al. 1999) separated by 90 mas. On the other hand, earlier MERLIN observations which did recover most of the $\mathrm{OH}$ single-dish flux (Montgomery \& Cohen 1992) indicated emission all the way between these two clumps with a velocity gradient. The authors interpreted this result as a rotating disk of $\mathrm{OH}$ with a dynamical enclosed mass of $\sim 10^{8} M_{\odot}$ within a $100 \mathrm{pc}$ radius. However, these observations were at low resolution $\left(0.3^{\prime \prime}\right)$, and so significant superresolution was required to detect the gradient. Trotter et al. (1997) suggested that the disk-like velocity gradient might be the result of blending between the two VLBI clumps when observed at the lower resolution of MERLIN.

Given the large amount of missing flux in the VLBI experiments it is, however, difficult to rule out the possibility of a rotating disk since more diffuse maser emission may well be distributed in a gravitationally bound disk. In order to check the result of Montgomery \& Cohen (1992) we have therefore performed simultaneous EVN and MERLIN observations of the $\mathrm{OH}$ masers in III Zw 35. Since the former MERLIN results were published the spatial resolution of MERLIN has been improved by a factor of two by the addition of a telescope at Cambridge. By including the baselines provided by EVN we were able to cover the baseline lengths up to those of the VLBA and global VLBI, so achieving a more complete picture of the maser emission.

The paper is organised as follows, in Sect. 2 we present the details of the observations and data reduction. The results of the line observations are presented in Sect. 3 while the continuum results are presented in Sect. 4 . The nature of the continuum and line emission is discussed in Sect. 5. Finally in Sect. 6 we summarise our results and the interrelationship between the line and continuum components, briefly compare III Zw 35 to other megamaser galaxies and consider the prospects for future observations of III Zw 35.

\section{Observations and data reduction}

In June 1998 we used 8 antennas from the European VLBI Network (Cambridge, Effelsberg, Lovell, Medicina, Noto, Onsala, Torun and Westerbork) to observe the two main $\mathrm{OH}$ maser transitions (1667 and $1665 \mathrm{MHz}$ ) in III Zw 35 (at velocity $V_{\mathrm{CO}}=8289 \mathrm{~km} \mathrm{~s}^{-1}$, Mirabel \& Sanders 1987). This EVN array provided a baseline range between 198-2280 km, corresponding to fringe spacings between 16-190 mas. Simultaneously, as we describe below, observations were made with the MERLIN array (in the $1667 \mathrm{MHz}$ transition only) which had baselines between 6.2 and $217 \mathrm{~km}$ (fringe spacings between $0.17^{\prime \prime}-5.8^{\prime \prime}$ ).

The EVN observations were conducted with dual circular polarisation. In order to achieve high spectral resolution for both $\mathrm{OH}$ transitions, two $4 \mathrm{MHz}$ bands were centred on the redshifted frequencies corresponding to $1665 \mathrm{MHz}$ and $1667 \mathrm{MHz}$ respectively. We also observed continuum emission in two additional $4 \mathrm{MHz}$ bands located on each side of the maser frequencies. The source was tracked for $12 \mathrm{hrs}$; repeated 44 min observing runs on source was intermixed with 22 min observing runs on the compact nearby calibrator $0133+476$. The data were correlated using the VLBA correlator with 512 channels yielding a channel separation of $1.5 \mathrm{~km} \mathrm{~s}^{-1}$, and were reduced within AIPS.

Data from Jodrell and Medicina were affected by RFI in single channels which could be edited out, however, the RFI affected the IF levels and $T_{\text {sys }}$ values. A priori amplitude calibration was done using $T_{\text {sys }}$ tables, and amplitude refinement via the selfcal procedure was later performed on the compact external calibrator $0133+476$ and applied to the target source. We are confident of the relative amplitude calibration between antennas but since the absolute flux of $0133+476$ is variable and unknown, the absolute flux scale may be in error by up to $10 \%$. Fringe-fitting and phase-calibration was done using data from the strongest 20 maser channels of the $1667 \mathrm{MHz}$ line. Fringe rate and phase solutions so derived were subsequently applied to all channels of the 1667 and $1665 \mathrm{MHz}$ data, as well as to the continuum. In constructing natural weighted cubes channels were averaged in pairs to give a final spectral line velocity resolution of $2.97 \mathrm{~km} \mathrm{~s}^{-1}$. Each channel map was restored with a $25 \times 21.2$ mas beam, and the resulting $1 \sigma$ noise in the $1667 \mathrm{MHz}$ cube was $1.6 \mathrm{mJy} /$ beam/channel. The $1665 \mathrm{MHz}$ dataset contained less data due to more serious RFI problems at this frequency, and so for the $1665 \mathrm{MHz}$ data the $1 \sigma$ noise was $2.9 \mathrm{mJy} /$ beam/channel. Continuum maps were produced using the two line free IFs $(8 \mathrm{MHz}$ in total) and gave a $1 \sigma$ noise of $0.33 \mathrm{mJy} /$ beam using uniform weighting, and $0.1 \mathrm{mJy} /$ beam for natural weighting.

Simultaneous dual polarisation observations were conducted with the MERLIN array. Only the $1667 \mathrm{MHz}$ line was observed, with a resulting velocity resolution of $11 \mathrm{~km} \mathrm{~s}^{-1}$. The data were reduced similarly to the EVN data, and the final cube has a $1 \sigma$ channel noise of $1.9 \mathrm{mJy} /$ beam. Each channel was restored with a beam of 
size $183.3 \times 131.8$ mas. When mapping the continuum, we used the line free channels within this IF. This continuum map has a $1 \sigma$ noise of $0.7 \mathrm{mJy} /$ beam.

In order to combine the EVN and MERLIN arrays, the EVN data were averaged in frequency to correspond to the MERLIN data. Both datasets were self-calibrated to the same channel peak, and concatenated to form one dataset. Two cubes were produced, one with natural weighting ( $1 \sigma \mathrm{rms} 1.6 \mathrm{mJy} / \mathrm{beam} / \mathrm{channel}$ and a restoring beam of size $43.3 \times 35.6$ mas) and the second with uniform weighting and tapered with a Gaussian of width 0.3 at $5 \mathrm{M} \lambda$ (giving $1 \sigma \mathrm{rms} 3.1 \mathrm{mJy} / \mathrm{beam} /$ channel and a restoring beam of size $33.0 \times 28.7$ mas).

\section{Results: $\mathrm{OH}$ maser emission and absorption}

\section{1. $1667 \mathrm{MHz}$ maser and absorption}

The spatial extent of the $1667 \mathrm{MHz}$ emission was obtained by selecting and frequency averaging the channels covering the $\mathrm{OH}$ maser emission for the combined EVN and MERLIN array. The resulting distribution of maser emission in Fig. 1 is dominated by two compact regions of maser emission separated by 88 mas $(44 \mathrm{pc})$ at a position angle of $-10^{\circ}$. Following the labeling of the different masing positions of Trotter et al. (1997), these two regions correspond to the Southern and Northern maser clumps, which were also detected by global VLBI (Diamond et al. 1999). However, in addition to these we also detect two arcs of weaker maser emission, containing gas with intermediate velocities. Note that in the centre of the map there is a region devoid of maser emission. This spatial distribution suggests a disk or torus-like structure. Assuming that such a structure would be circular, we estimate that the plane of the disk is inclined at an angle of $\sim 60^{\circ}$ to the sky plane. In our shortest MERLIN baseline, we find a peak flux of $200 \mathrm{mJy}$, which suggests that we are recovering $80 \%$ of the total single-dish flux. Hence, we are able to recover a larger part of the maser flux than what was possible in earlier VLBI and VLBA observations.

By excluding regions of low SNR in the naturally weighted EVN + MERLIN cube (which will blank out the weaker Eastern part of the ring) we plot in Fig. 2 the iso-velocity contours across the maser ring. This plot displays a general velocity gradient of $\sim 1.5 \mathrm{~km} \mathrm{~s}^{-1} \mathrm{pc}^{-1}$ from the South to the North, including the regions of diffuse maser emission. This is consistent with the compact masers and the diffuse masers being part of a slowly rotating disk. However, the velocity contours in the South appear distorted.

Using the EVN-only data gives higher spatial resolution, and these results can be viewed in Fig. 3. By using the AIPS task ISPEC we take spectra at different spatial positions as marked in the inset map (Fig. 3). At positions 5 and 7 (corresponding to the Northern and Southern maser clumps respectively) there is strong maser emission, and the arc-shaped emission can be seen at positions 11 and 12 . In addition there is a hint of maser emission at

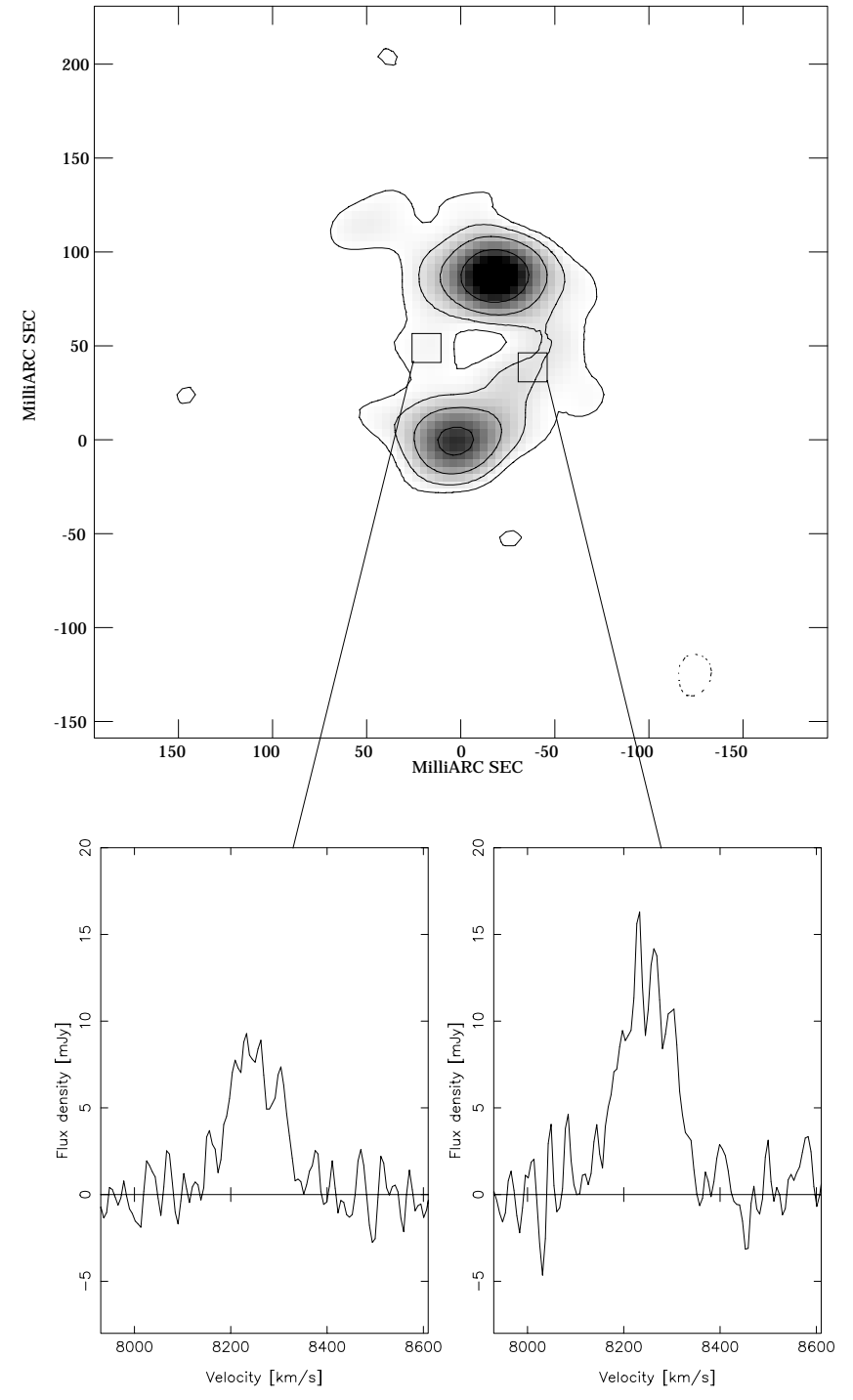

Fig. 1. Uniformly weighted map of the integrated $1667 \mathrm{MHz}$ maser emission for the EVN and MERLIN combined array. The data has been averaged in frequency over all spectral channels showing $\mathrm{OH}$ maser emission. The data has been tapered by a Gaussian of value 0.3 at $5 \mathrm{M} \lambda$ and the beam size is $33.0 \times$ 28.7 mas. Plotted contours are $-1,1,2,4$ and 8 times the $3 \sigma \mathrm{rms}$ noise of $2.7 \mathrm{mJy} /$ beam. Bottom panels show spectra taken at the positions indicated by the boxes. Note that there is a region in the centre devoid of maser emission.

position 2 (due to the higher resolution compared to Fig. 1 we resolve out most of the weaker emission on the Eastern side of the ring). In addition to the maser emission at some positions we also see $\mathrm{OH}$ absorption. Following a ridge-line along the major axis of the source through the Northern and Southern maser clumps, $\mathrm{OH}$ absorption can be seen at positions $3,4,8,9$ and possibly at 6 . The shape of the absorption line is complex, and is most likely an effect of overlaid weaker maser emission features. Due to this contamination of the absorption line it is difficult to determine a centroid velocity, however, the overall trend from 3-9 indicates a velocity gradient with lower velocities 


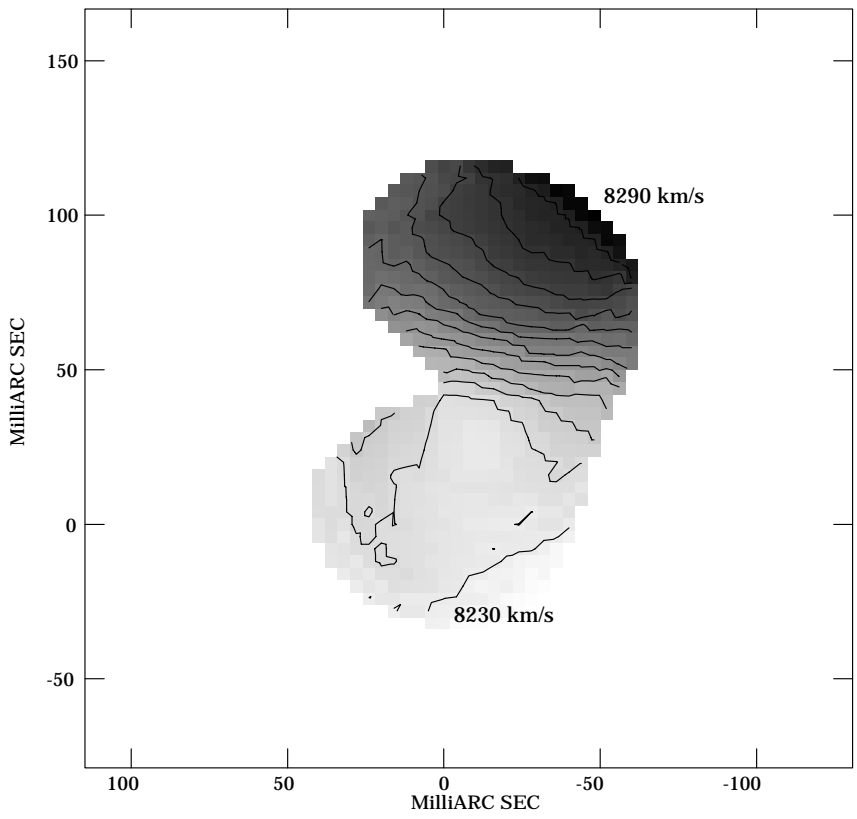

Fig. 2. The $\mathrm{OH}$ maser velocity field from the $\mathrm{EVN}$ and MERLIN combined arrays. Grey-scale is between 8224 and $8300 \mathrm{~km} \mathrm{~s}^{-1}$. Contours are from $8230 \mathrm{~km} \mathrm{~s}^{-1}$ and increasing by $5 \mathrm{~km} \mathrm{~s}^{-1}$ up to $8290 \mathrm{~km} \mathrm{~s}^{-1}$. Beam size $43.3 \times 35.6$ mas. At this resolution the weaker Eastern side is blanked out due to low SNR.

in the South. In total we find signs of $\mathrm{OH}$ molecules in a disk-like structure of radius of $\gtrsim 75 \mathrm{pc}$.

By comparing the continuum flux density mapped with the combined MERLIN + EVN data (see Sect. 4), we are able to estimate the opacity of the $\mathrm{OH}$. The $\mathrm{OH}$ linewings suggest the lines are not saturated. Comparing the absorption line and continuum emission in box 4 of Fig. 3 we estimate the peak opacity to be around 2, which for the observed $F W H M$ linewidth of $\sim 110 \mathrm{~km} \mathrm{~s}^{-1}$ yields an $\mathrm{OH}$ column density $N_{\mathrm{OH}}=5 \times 10^{16} T_{\mathrm{ex}} \mathrm{cm}^{-2}$.

It is also worthy of note, that in our images constructed from EVN-only data (see Fig. 3) we resolve out the continuum but still see absorption. That is to say in the spectra showing absorption the continuum levels are close to zero and the absorption extends to negative flux densities (note the data have not had continuum subtraction applied). This result indicates that the $\mathrm{OH}$ absorbing gas is distributed in a more compact spatial structure than the continuum. The data are consistent with for instance a band of high opacity absorption of width of order 20 mas extending outward along the major axis from the Northern and Southern maser clumps respectively and crossing a much more uniform continuum structure. As discussed in Sect. 5 this is consistent with what would be expected if the gas is in a high inclination disk with maser emission at small radii and absorption at larger radii.

From Fig. 3 we estimate the $\mathrm{OH}$ disk has a total diameter of 300 mas, or 150 pc. This size must be a lower limit since the detection of $\mathrm{OH}$ absorption will be hampered by a decreasing continuum level at larger radii. We find in the $\mathrm{OH}$ maser disk of III Zw 35 a velocity gradient of $\sim 1.5 \mathrm{~km} \mathrm{~s}^{-1} \mathrm{pc}^{-1}$. Assuming that the compact masers lie at the tangent points of the rotating ring, we use their velocity and position offset $\left(65 \mathrm{~km} \mathrm{~s}^{-1}\right.$ and $44 \mathrm{pc}$ respectively) and the derived disk inclination of $60^{\circ}$ to estimate the enclosed mass. We find the dynamical mass within a radius of 22 pc to be $M \sim 7 \times 10^{6} M_{\odot}$. Sanders et al. (1991) use their single dish $\mathrm{CO}(1 \rightarrow 0)$ observations to derive a total molecular mass of $6.9 \times 10^{9} M_{\odot}$ in III Zw 35 . This considerably exceeds our derived value within $r \simeq 22 \mathrm{pc}$ which implies that most of the molecular gas lies outside this radius.

\section{2. $1665 \mathrm{MHz}$ maser}

We also detected emission from the $1665 \mathrm{MHz}$ line in our EVN data (Fig. 4). While it is considerably weaker than the $1667 \mathrm{MHz}$ emission, the emission is spatially coincident with the two main regions of $1667 \mathrm{MHz}$ maser emission. The velocity agrees well with the velocity of the $1667 \mathrm{MHz}$ line. This is consistent with the observations by Diamond et al. (1999) who found one weak $1665 \mathrm{MHz}$ maser feature towards the Southern clump. No diffuse $1665 \mathrm{MHz}$ emission was detected between these positions. Since a large part of the $\sim 25 \mathrm{mJy} 1665 \mathrm{MHz}$ single-dish flux (Staveley-Smith et al. 1987) is missing, it is possible that its distribution follows the diffuse $1667 \mathrm{MHz}$ emission in the ring. However, it falls below our detection limits.

An estimate of the flux ratio of the 1667 and $1665 \mathrm{MHz}$ masers yields $F_{1667} / F_{1665} \sim 6$ for the Northern clump and $\sim 8$ for the Southern clump (Table 1 ). Given the noise levels of our spectral cubes (see Sect. 2) and the error from reading the levels from published total power spectra, these numbers are comparable. By adopting a $3 \sigma$ non-detection limit assuming a linewidth of $\sim 150 \mathrm{~km} \mathrm{~s}^{-1}$ we set a lower limit to the diffuse $F_{1667} / F_{1665}$ ratio of 5 . Another estimate of the diffuse line ratio can be achieved by subtracting the compact maser flux from the total flux in the single-dish spectra (Staveley-Smith et al. 1987). Although the error bars are quite large due to the uncertainties in the cubes, the flux ratio does not vary significantly for the diffuse and compact maser emission. This consistency can be explained if both diffuse and compact masers are pumped with similar rates and have the same population inversion of the molecular energy levels.

The high ratio of the $1667 / 1665 \mathrm{MHz}$ line is, given the uncertainties in the spectral cubes, consistent with the ratio of 9 observed by Mirabel \& Sanders (1987). They argued the high ratio was due to a high pumping efficiency (a large $F_{1667} / F_{60 \mu \mathrm{m}}$ ratio). We can rule out LTE conditions, which would require a $F_{1667} / F_{1665}$ ratio of 1.8 . Yates et al. (2000) draw a similar conclusion for the 1667 and $1665 \mathrm{MHz}$ transitions in Mrk273, which however show a more modest ratio of 5.5 .

\section{Results: $18 \mathrm{~cm}$ continuum}

In Fig. 5 we plot the distribution of the continuum emission at different resolutions. In all images the contours 

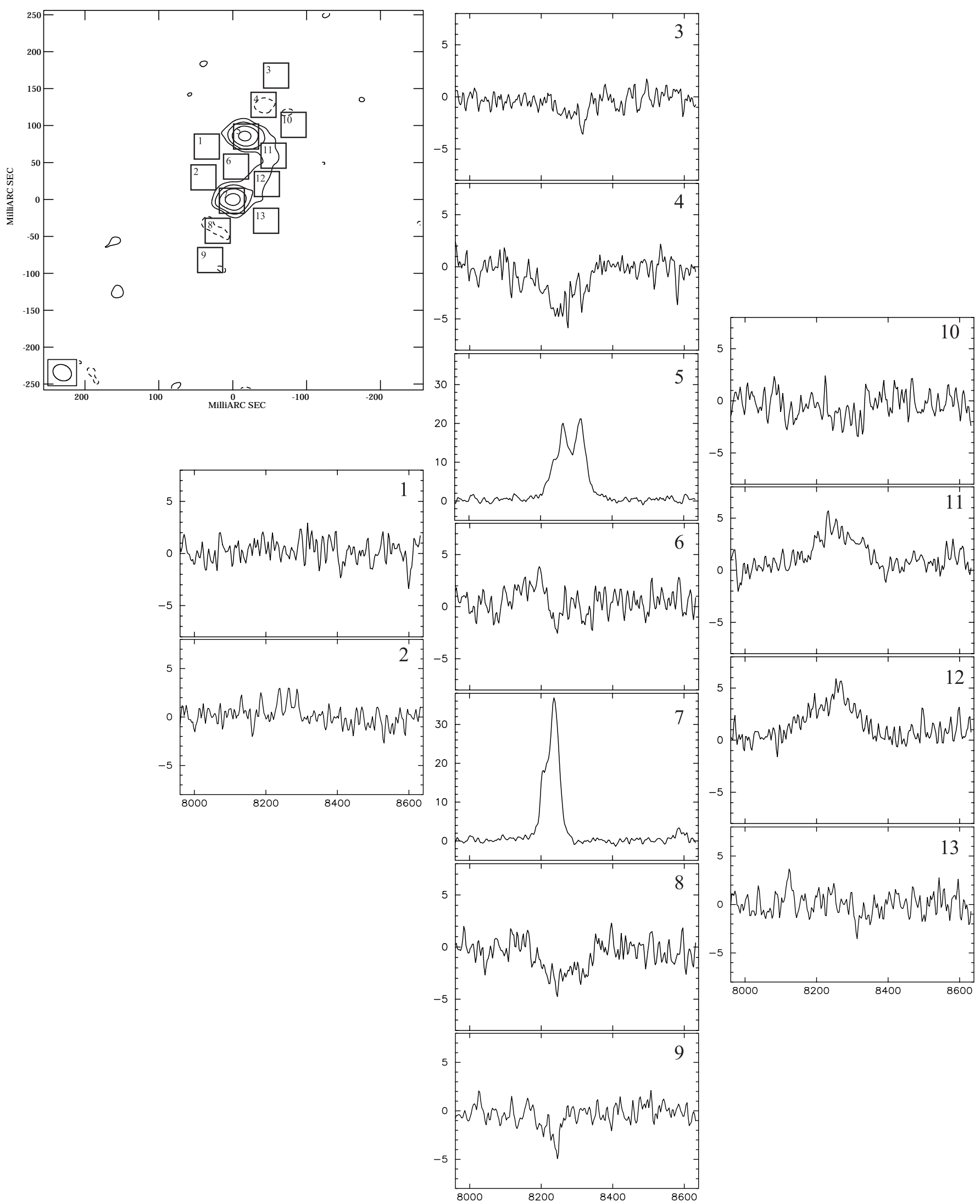

Fig. 3. The spatial distribution of $\mathrm{OH}$ maser emission and absorption. The inset map in top left corner is the EVN data averaged over all channels showing $1667 \mathrm{MHz}$ maser emission. Plotted contours are $-1,1,2,4$ and 8 times $2.0 \mathrm{mJy} / \mathrm{beam}$. The negative contours next to the emission indicate absorption (see Sect. 3.1). The restoring beam is $26.0 \times 21.2$ mas, at this resolution we resolve out the weaker Eastern side of the $\mathrm{OH}$ maser ring (cf. Fig. 1). Boxes on the EVN map represent regions over which spectra were taken. These spectra are plotted in flux density (mJy) versus heliocentric velocity $\left(\mathrm{km} \mathrm{s}^{-1}\right)$. 
Table 1. The $1667 \mathrm{MHz}$ and $1665 \mathrm{MHz}$ peak fluxes at different positions and resolutions, in addition to the corresponding $1667 / 1665 \mathrm{MHz}$ flux density ratios.

\begin{tabular}{lccc}
\hline \hline Position & $\begin{array}{c}F_{1667} \\
(\mathrm{mJy})\end{array}$ & $\begin{array}{c}F_{1665} \\
(\mathrm{mJy})\end{array}$ & $F_{1667} / F_{1665}$ \\
\hline Compact N & 46 & 7.5 & 6 \\
Compact S & 66 & 8.2 & 8 \\
Total $^{1} \mathrm{~N}$ & 150 & 21 & 7 \\
Total $^{1} \mathrm{~S}$ & 258 & 33 & 8 \\
Diffuse $^{2} \mathrm{~N}$ & 104 & 13.5 & 8 \\
Diffuse $^{2} \mathrm{~S}$ & 192 & 24.8 & 8 \\
\hline
\end{tabular}

${ }^{1}$ Estimated from the single-dish spectra presented by StaveleySmith et al. (1987), assuming the flux density at $\sim 8245 \mathrm{~km} \mathrm{~s}^{-1}$ arises in the Northern clump and the flux density at $\sim 8310 \mathrm{~km} \mathrm{~s}^{-1}$ in the Southern clump.

${ }^{2}$ Estimated total diffuse maser contribution obtained by subtracting the compact $\mathrm{N}$ and $\mathrm{S}$ flux densities in our maps (on the two top rows) from the single dish total flux densities.

represent the continuum, and the grey scale plots the position of the integrated maser emission. Using the MERLIN data only (upper left panel) we detect a total flux density of $38 \mathrm{mJy}$ which is consistent with the flux earlier observed at the VLA and at MERLIN (Chapman et al. 1990). In the upper right panel we plot the combined EVN and MERLIN data, where we pick up $32 \mathrm{mJy}$ in the CLEAN components. The lower left panel in Fig. 5 plots the naturally weighted EVN-only continuum. This map shows what appear to be 5 or 6 distinct peaks of emission. A total continuum flux density of $18 \mathrm{mJy}$ is recovered in the CLEAN components. Increasing the resolution even further (uniform weighting, bottom right panel in Fig. 5) the continuum appears to consist of a few compact sources, together producing a total flux of $\sim 5 \mathrm{mJy}$ ( $13 \%$ of the total emission).

Note in making the images in Fig. 5 we cleaned data which was phase-calibrated using the strong $\mathrm{OH}$ maser images; therefore no self-calibration was applied to the continuum data. Furthermore no boxes were used in the CLEAN to artificially constrain where the continuum should lie. We are therefore confident of the reliability of the main features in the three lowest resolution images shown in Fig. 5. However for the highest resolution, uniformly weighted EVN image the signal to noise is low (the highest peak is only at $4 \sigma$ ) and we find that the relative brightness of intensity peaks changes depending on the grid size used. This is presumably because the grid size effects the details of the data weighting when uniform weight option is chosen. This variability in structure indicates that this image is less reliable then the others. In the version of the image given in Fig. 5d there are 6 features above $4 \sigma$ and no feature below $-4 \sigma$. At several such $4 \sigma$ positions there are corresponding $6 \sigma$ detections in the lower resolution image Fig. $5 \mathrm{c}$. We suspect these to be real sources. However, given the sensitivity of the present data we cannot be absolutely sure of the reality of each indi-

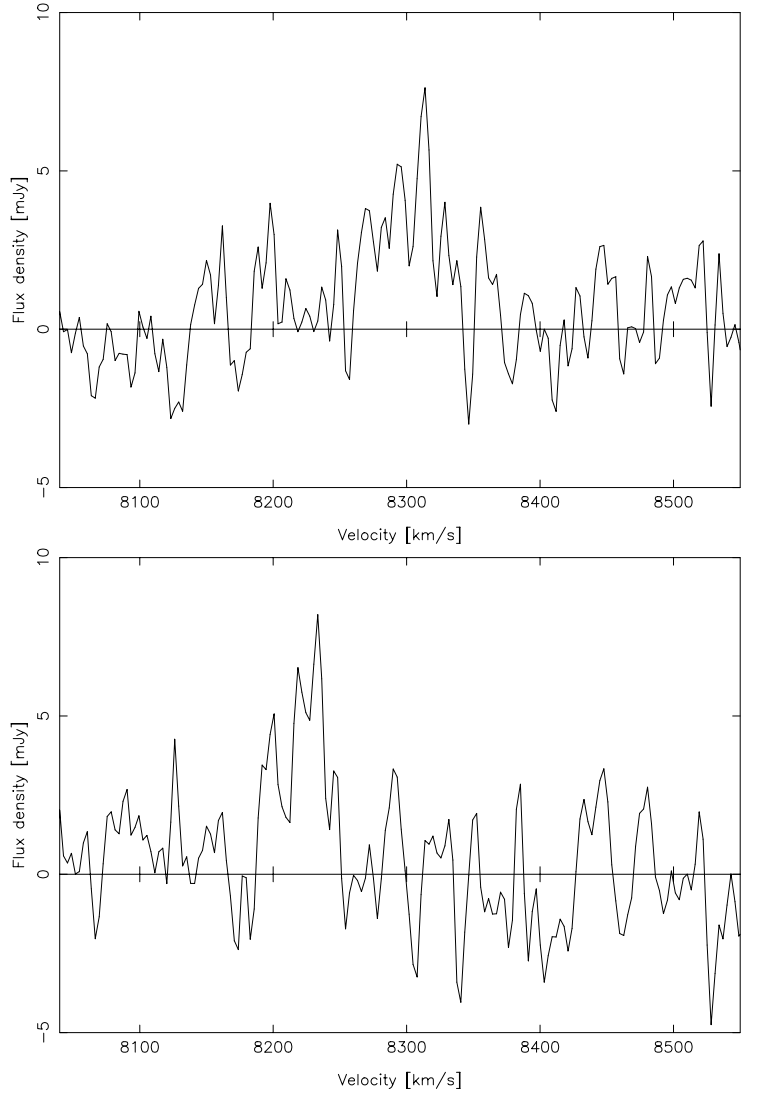

Fig. 4. Spectra of the maser emission seen at $1665 \mathrm{MHz}$. Top: over the Northern maser clump. Bottom: over the Southern maser clump. We are not sensitive to any diffuse $1665 \mathrm{MHz}$ emission that presumably follows the distribution of the $1667 \mathrm{MHz}$ maser ring.

vidual component, and therefore we do not give a table of fluxes and positions of these individual features.

The images presented in Fig. 5 show that the continuum emission in III Zw 35 consists of two parts, one of which is compact but perhaps just resolved by our VLBI observations. The second part is diffuse resolved emission. Such a division into two components is also clearly seen in our amplitude versus $u, v$-distance plots. From these plots we see that the diffuse component has a FWHM size of $\sim 460$ mas $(230 \mathrm{pc})$ and a flux density $\gtrsim 32 \mathrm{mJy}$. The corresponding peak brightness temperature is $1 \times 10^{5} \mathrm{~K}$. The size and brightness temperatures of the compact components are hard to measure given their faintness. However, for every component A-E with flux exceeding $6 \sigma$ in Fig. $5 \mathrm{c}$ we perform Gaussian fitting using the AIPS task JMFIT. While each of the components A, B, C and D were fitted with one Gaussian, we allowed two Gaussians over component E. The resulting sizes of the fitted Gaussians are similar within a factor of 1.5 , with an average size of 30 mas $(15 \mathrm{pc})$. The corresponding average brightness temperature is of the order $7 \times 10^{5} \mathrm{~K}$ (though this must be confirmed by future higher sensitivity observations). Our uniformly weighted image (resolution $18 \times 12$ mas) detected a few of these components at a 3 or $4 \sigma$ level. The noise in this image is a factor 3 larger than in the 

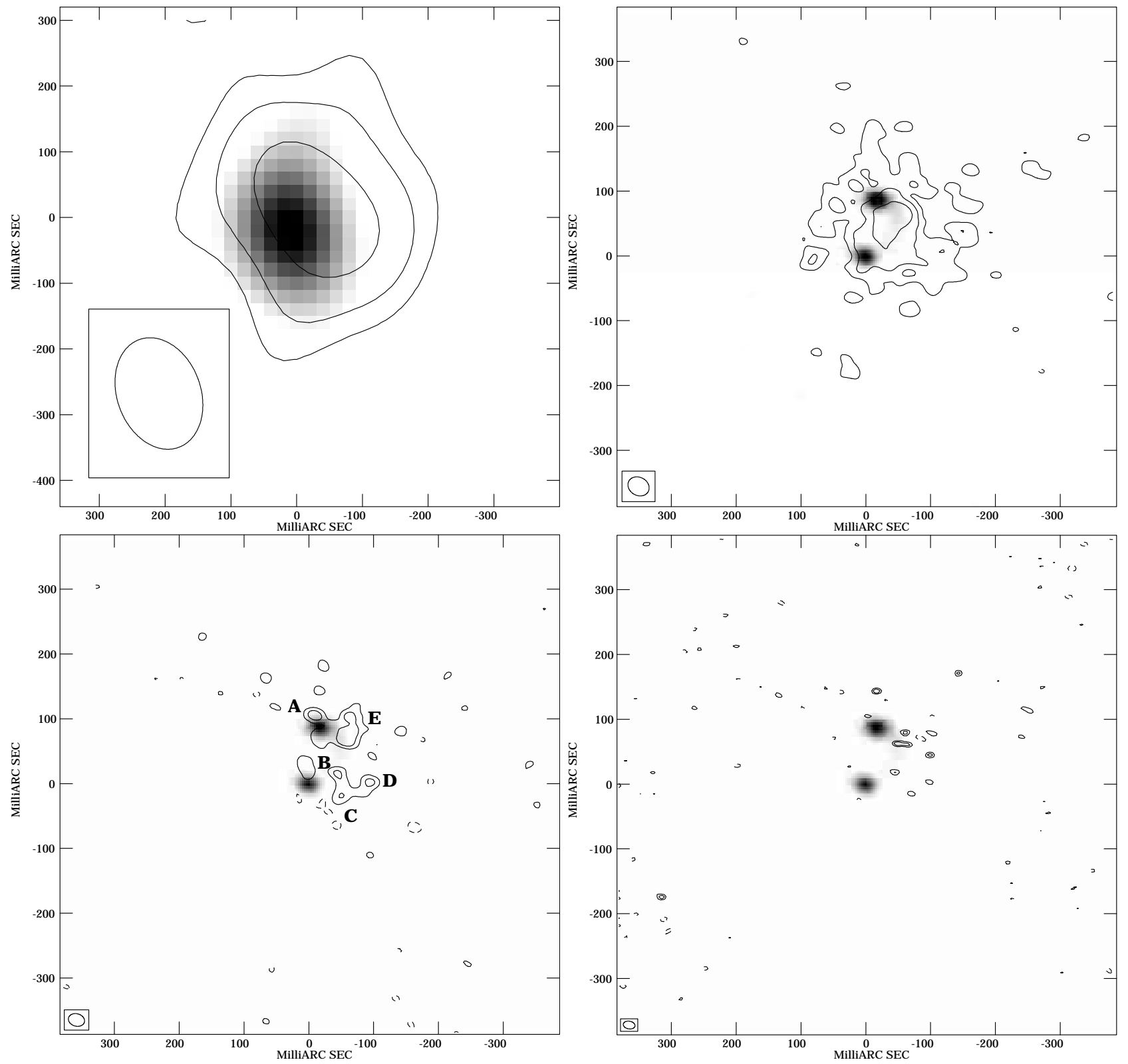

Fig. 5. The continuum distribution in III Zw 35 . These maps display with different resolutions the continuum in contours and the $1667 \mathrm{MHz}$ maser emission superposed in grey-scale. Beam sizes are shown in the lower left corner of each image. Increasing the resolution the continuum appears to resolve into a number of compact sources. Top left: MERLIN-only map at a resolution of $174 \times 127$ mas. Plotted contours are $-1,1,2$ and 4 times the $3 \sigma$ rms noise of $2.1 \mathrm{mJy} /$ beam. Note that this map has not the same phase centre as of the three other maps which contain EVN data. Top right: EVN + MERLIN combined continuum at a resolution of $34 \times 28$ mas, plotted contours are $-1,1,2$ and 4 times the $3 \sigma$ rms noise of $0.27 \mathrm{mJy} /$ beam. Bottom left: EVN-only naturally weighted map, resolution $25.4 \times 19.2$ mas. Plotted contours are $-3,3$ and 6 times the $1 \sigma$ rms noise of $0.1 \mathrm{mJy} / \mathrm{beam}$. Bottom right: EVN-only uniform weighting, resolution $18 \times 11.7$ mas. Plotted contours are $-3,3$ and 4 times the $1 \sigma$ rms noise of $0.33 \mathrm{mJy} /$ beam. Note the appearance of this highest resolution image depended somewhat on the choice of grid spacing as described in the text, which in addition to the low dynamic range makes it less reliable than the other maps.

naturally weighted map which partly explains the lower detection rate, but may also be due to some fraction of the emission being resolved out in going to higher resolution. Such resolution effects are consistent with relatively large sized components (30 mas) explaining why the total recovered flux density decreases from $18 \mathrm{mJy}$ in Fig. 5c to $5 \mathrm{mJy}$ in Fig. 5d. On the other hand, the data could also be consistent with smaller discrete sources ( $<10$ mas) superimposed on more diffuse emission which is resolved out when the resolution is increased from that in Figs. 5c to $5 \mathrm{~d}$.

We note that the continuum was not detected in the VLBA observations by Trotter et al. (1997) who achieved a theoretical noise in a natural weighted image of $0.2 \mathrm{mJy} /$ beam (resolution $25 \times 12 \mathrm{mas}$ ). The sensitivity of our EVN map in terms of flux density per beam is better by a factor of 2 , and these observation have a beam area almost twice as large as for the VLBA observations. 
Given these differences we conclude that there is no discrepancy between our detection of the $18 \mathrm{~cm}$ continuum and the non-detection reported by Trotter et al. (1997), especially if parts of this emission is partly resolved by the smaller beam of the VLBA observation. An additional factor in considering previous continuum non-detections is the possibility that the emission is variable, and that new features have appeared since the earlier global VLBI and VLBA observations. This is plausible if the compact components are supernovae created with the rate we estimate in Sect. 5.1.1.

\section{Discussion}

In this section we discuss first the nature of the continuum emission that we have detected (see Sect. 5.1) and then discuss the origin of the megamaser emission (see Sect. 5.2). Since we intend to write a follow up paper investigating in more detail the geometry and physical conditions of the $\mathrm{OH}$ megamaser emission, the discussion in Sect. 5.2 will be relatively brief. Finally in Sect. 6 we summarise our results, compare III Zw 35 to other megamaser galaxies and discuss prospects for future observations.

\subsection{The origin of the radio continuum emission}

Both AGN and starbursts have been candidates as the power source for the far-infrared luminosities of LIRGs. Chapman et al. (1990) claimed the Northern nucleus of III Zw 35 to be a LINER, mainly based on optical spectroscopy. On the other hand different results have been obtained by other groups performing spectroscopic identifications of LIRGs (Smith et al. 1998b [SLL98]; Veilleux et al. 1995), classifying III Zw 35 as a starburst. There are several additional arguments supporting the existence of a starburst in III Zw 35, for instance the high ratio $L_{\text {ir }} / M\left(\mathrm{H}_{2}\right) \sim 51.3$ (Sanders et al. 1991) which could indicate an increased star formation efficiency. III Zw 35 also appears to lie on the well-known radio-IR correlation for starbursts (e.g. Helou et al. 1985). This correlation can be quantified by measuring the ratio $\log \left(S_{60 \mu \mathrm{m}} / S_{\mathrm{t}+\mathrm{nt}}\right)$ where $S_{60 \mu \mathrm{m}}$ is the $60 \mu \mathrm{m}$ flux density and $S_{\mathrm{t}+\mathrm{nt}}$ includes both thermal and non-thermal $1.4 \mathrm{GHz}$ flux density. This ratio equals $2.15 \pm 0.3$ for infrared selected galaxies (Condon \& Broderick 1988). A value less than 1.6 would indicate an AGN, since there would be an excess of non-thermal continuum as compared to starburst galaxies. For III Zw 35 we estimate this ratio to be 2.47 which is consistent with a starburst model. Finally, at $25 \mu \mathrm{m}$ the $I R A S$ flux is $1 \mathrm{Jy}$, while it is $\leq 0.1$ Jy at $12 \mu \mathrm{m}$ implying a red IR colour which is more consistent with dust heated by a starburst rather than an AGN.

In the continuum image there is no sign of an AGN component, therefore we test in Sect. 5.1.1 the possibility that most of the total radio flux density (the diffuse component) derives from overlapping emission due to supernova remnants (SNRs). For the compact continuum components, we can rule out any thermal origin since the brightness temperatures exceeds $7 \times 10^{5} \mathrm{~K}$. Instead we show in Sect. 5.1.2 that these individual compact continuum features may well be attributed to individual radio supernovae (RSNe) or clusters of RSNe or young SNRs.

\subsubsection{Resolved continuum due to emission from supernova remnants}

For galaxies exhibiting modest radio luminosities, the tight correlation between the $1.4 \mathrm{GHz}$ radio luminosity and the FIR luminosity is generally explained by formation of massive stars which both heats the dust as well as producing synchrotron radiation through supernova remnants. Following the discussion in the paper by Condon (1992), the FIR flux of III Zw $35\left(\log (F I R)=11.3 L_{\odot}\right)$ can be used to estimate the SFR to $19 M_{\odot} \mathrm{yr}^{-1}$, with a corresponding supernovae rate $\nu_{\mathrm{SN}} \simeq 0.8 \mathrm{yr}^{-1}$. Further, such a supernovae rate would produce a non-thermal luminosity of $7.1 \times 10^{22} \mathrm{~W} \mathrm{~Hz}^{-1}$. The observed value is $5.8 \times 10^{22} \mathrm{~W} \mathrm{~Hz}^{-1}$, and so it appears that the diffuse $18 \mathrm{~cm}$ continuum radiation can readily be explained by a starburst.

\subsubsection{Nature of compact components}

Although the dominant part of the radio emission can be explained by radiation due to old supernova remnants (ages $\geq 100 \mathrm{yr}$ ), we also observe (see Sect. 4) compact radio components. One possibility is that they are individual radio supernovae. The compact features in III Zw 35 show typical $18 \mathrm{~cm}$ luminosities of $\sim 10^{21} \mathrm{~W} \mathrm{~Hz}^{-1}$, which are similar to those of the objects observed in Arp 220 (a few $\times 10^{20} \mathrm{~W} \mathrm{~Hz}^{-1}$; Smith et al. 1998a) and in Mrk $273(2.5 \times$ $10^{21} \mathrm{~W} \mathrm{~Hz}^{-1}$, Carilli \& Taylor 2000). Such luminosities are much larger than typical RSN luminosities $\left(L_{5 \mathrm{GHz}} \sim\right.$ $10^{17} \mathrm{~W} \mathrm{~Hz}^{-1}$; Panagia et al. 2001). On the other hand, such luminosities are comparable to those of the brightest RSN detected, for instance SN1986J which peaked in its $20 \mathrm{~cm}$ luminosity at a value of $2.5 \times 10^{21} \mathrm{~W} \mathrm{~Hz}^{-1}$ (Weiler et al. 1990). Smith et al. (1998a) suggest that the Arp 220 objects are such highly luminous radio supernovae. Pedlar (2000) on the other hand proposes that they could be young supernova remnants, since the Arp 220 objects fit on an anti-correlation between known SNR source size and radio luminosity. Returning to III Zw 35 , we cannot rule out the possibility that the compact objects we detect are single, extremely luminous radio supernovae.

Generally we can assume that if we detect individual radio supernovae they must be in their strongest phase and therefore must have burst very recently. Thereafter they will dim while expanding and result in a lower surface brightness to which our observations are insensitive. Later on, when the ejecta interact with the dense ISM they will brighten and contribute to the diffuse radio emission in their SNR phase (see Sect. 5.1.1). SLL98 derive an 
expression for the light curve of a radio supernova (RSN) after it has reached its maximum luminosity:

$$
L_{\nu}=L_{\nu, \max }\left(\frac{\Delta t}{3 \mathrm{yr}}\right)^{\beta}
$$

Following their discussion, we assume that we are dealing with luminous $\mathrm{RSN}$ and adopt $L_{1.6 \mathrm{GHz} \text { max }} \simeq 1.4 \times$ $10^{21} \mathrm{~W} \mathrm{~Hz}^{-1}$ and $\beta=-1.3$. Given these assumptions individual RSN will lie above our detection level for 7 years. Given our adopted supernova rate of $0.8 \mathrm{yr}^{-1}$ we thus expect roughly 6 single RSN above our sensitivity level in our naturally weighted map, which is consistent with what we observe.

The first-order estimates given from the discussion above imply that more or less all supernovae would have to be of the extremely luminous type. An alternative explanation for the large luminosities of each compact feature is that they might consist of several RSN or young SNRs superimposed. Adopting a minimum radio emission producing period of $\sim 2 \times 10^{4}$ yr (e.g. Condon \& Yin 1990) for each SNR at $\lambda=18 \mathrm{~cm}$, then given our inferred supernova rate of $0.8 \mathrm{yr}^{-1}$ we expect at least 16000 supernova remnants within the starburst region. Given that we have radio emission over a total size of $340 \times 430$ mas (corresponding to $677 \mathrm{EVN}$ beams), assuming an uniform distribution of SNRs we would expect to see around 24 SNRs within each beam. Or equivalently, each beam should contain a luminosity $\gtrsim 10^{20} \mathrm{~W} \mathrm{~Hz}^{-1}$. This is an order of magnitude lower than we see in our compact objects, but is sufficient to explain the diffuse emission as described in Sect. 5.1.1. However it is likely that the SNRs are distributed in clumps, resulting in more SNRs per compact feature. For instance, Meurer et al. (1995) imaged a sample of starburst galaxies with the HST and found that the starbursts are very irregular in their morphology. This HST data also suggests that clustering is important, with superstar clusters forming in the most central regions of the starbursts. These superstar clusters have sizes of from a few parsecs up to $10 \mathrm{pc}$, which could be consistent with the sizes indicated for our compact components (see Sect. 4). SSL98 fitted starburst models to a number of LIRGs detected by VLBI, and while a Gaussian, randomly detonating model fitted the data poorly a clustered model was more successful. In their cluster model the typical clump had a size of around 4 pc (which would be less than our VLBI beam of $9 \times 6 \mathrm{pc}$ ) and contained $8 \mathrm{RSN}$. However, it was assumed that all RSN within one clump detonated simultaneously.

A test could decide between the two possible scenarios for the compact continuum. By performing multi-epoch imaging of the compact components we could investigate if the distribution alters significantly over a period of 510 years, as is expected for a young and luminous population. If not, perhaps the compact components are better explained by clusters of supernova remnants.

\section{2. $\mathrm{OH}$ megamasers}

We hope in a subsequent paper to model in detail the kinematics, geometry and physical conditions of the $\mathrm{OH}$ maser emitting region. This modelling will require detailed simulation of the maser emission and propagation. It would also probably be useful to reanalyse existing global VLBI (Diamond et al. 1999) and VLBA data (Trotter et al. 1997) in light of our new results. In this present paper we confine ourselves to some general observations.

The most striking properties of the newly detected diffuse $\mathrm{OH}$ maser emission is its distribution in an apparent ring structure (see Fig. 1 and Sect. 3.1). The kinematic data (Fig. 2) is also consistent with a rotating ring, although there are distortions of the velocity field in the South. The Western side of the ring appears somewhat stronger than the Eastern side (Fig. 1), but this difference, as for similar differences in the continuum might be explained by free-free absorption effects (see Sect. 5.3), or to a weaker background continuum to amplify. Fig. $6 \mathrm{a}$ shows a possible geometry for the ring, while Fig. 6b shows the total path length through the ring. The ring is inclined at $60^{\circ}$ and has an outer radius of about $25 \mathrm{pc}$ and an inner radius of $16 \mathrm{pc}$, and the full thickness of the ring is also $16 \mathrm{pc}$. The ring is therefore relatively thick in the direction perpendicular to the plane of the ring. Such a large thickness is broadly consistent with the large $\mathrm{OH}$ velocity dispersions observed (see Fig. 3) which are comparable or larger than the inferred orbital velocity of $38 \mathrm{~km} \mathrm{~s}^{-1}$. Given the high velocity tails observed in the emission and absorption spectra it is possible that some fraction of the observed $\mathrm{OH}$ gas has reached escape velocity; accelerated out of the disk by supernovae explosions. It is possible therefore that we only see maser emission from gas in the midplane where densities and pumping conditions are suitable for maser action.

In Fig. 6c we show a model $\mathrm{OH}$ maser brightness distribution at the resolution of our EVN + MERLIN observations. In calculating this model brightness we assume that the masing $\mathrm{OH}$ exists within small clouds of volume filling factor 0.07 , and diameter $1 \mathrm{pc}$. We make the simplest possible assumptions consistent with the standard model of $\mathrm{OH}$ megamasers that the inverted $\mathrm{OH}$ amplifies seed photons from a continuum background of uniform brightness, and that all the masers are unsaturated. We further assume, as indicated by the data, that random velocity dispersions of the clouds are relatively large compared to the rotational velocity. This means that along all lines of sight systemic changes in velocity due to rotation are small compared to random cloud velocity dispersions. The brightness distribution shown in Fig. $6 \mathrm{c}$ is then calculated for a single velocity channel near the systemic velocity. Clouds at this radial velocity are randomly distributed within the volume of the annulus, the amplified continuum is calculated, and then this is convolved down to the same resolution as our observations.

Given the simplicity of the above model (see Figs. 6a,b) the match to the observations (see Figs. 1 and 2) is 

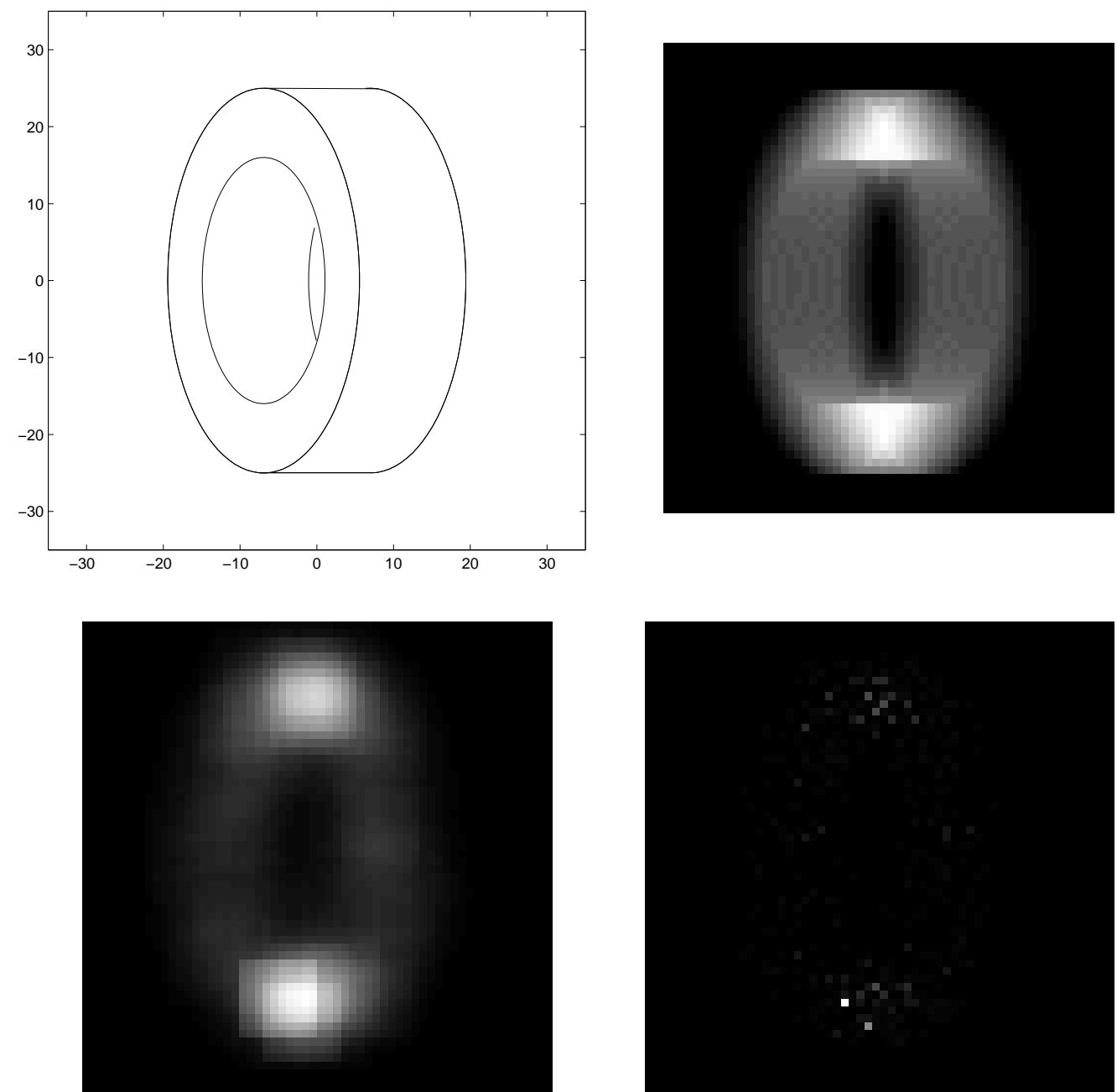

Fig. 6. a) Possible model of an inclined annulus at an inclination of $60^{\circ}$; inner and outer radius of annulus are $16 \mathrm{pc}$ and $25 \mathrm{pc}$ respectively. Annulus thickness is 16 pc. Large path lengths through the annulus occur at the North and South tangent points. b) Path length through inclined ring. c) Modeled $\mathrm{OH}$ maser brightness distribution at the resolution of the MERLIN+EVN observations (see text). The annulus is considered to be filled with clouds of diameter $1 \mathrm{pc}$, with volume filling factor 0.07 and opacity $\tau=-0.6$. The gray scale is linear, with the peak brightness shown as white. d) The same model plotted at full resolution, the North and South emission breaks up into individual bright spots, consistent with observations.

striking. At the front and back parts of the ring the path lengths through the annulus are small and the cloud covering factor within the beam is less than one. In these regions the apparent brightness is weak and is linearly dependant on the path length. At the tangent points however the path length is 3 or 4 times larger (see Fig. 6b) and so there is a significant chance of getting two or three clouds superimposed and hence strong maser emission. At the EVN+MERLIN resolution (see Fig. 6c) this gives bright regions at the tangent points. At higher resolution (see Fig. 6d) compact masers are detected at the positions where clouds overlap. Some Monte-Carlo realisations such as that shown in Fig. 6d give distributions of compact masers similar in structure to those observed by Trotter et al. (1997) and Diamond et al. (1999).

Despite the apparent success of the above model more detailed work is required to see whether it can fit all the details of the compact masers. In particular Diamond et al. (1999) detected a velocity gradient of $32 \mathrm{~km} \mathrm{~s}^{-1} \mathrm{pc}^{-1}$ over approximately $5 \mathrm{pc}$ in a subregion of the Northern compact masers. It may however be possible to explain such a gradient naturally in terms of clouds which partially overlap in both their projected positions and their radial velocities. If subsequent work shows the model described above to be essentially correct then its allows the exciting possibility of strongly constraining the sizes, filling factors and opacities of the $\mathrm{OH}$ masing clouds. Given these parameters detailed modelling of the maser emission intensity and line ratios such as attempted by Randell et al. (1995) will then be possible. We defer this detailed geometrical and physical modelling to a later paper.

\subsection{Source asymmetry due to free-free absorption?}

The distribution of the continuum emission is asymmetric, with most of the milli-arcsecond flux detected at the North-West side of the $\mathrm{OH}$ ring. In addition, the maser emission on the Eastern side is weaker than on the Western 
side. One hypothesis that could explain these observations is obscuration by a free-free absorbing medium. Given that there is a difference in the flux density of the $\mathrm{OH}$ masers of a factor of 2 between the two sides, we estimate the opacity of the absorbing medium to be $\tau \simeq 0.7$. Since this occurs at $1.6 \mathrm{GHz}$ it implies for a gas with electron temperature $10^{4} \mathrm{~K}$ an emission measure of $5.2 \times 10^{6} \mathrm{~cm}^{-6} \mathrm{pc}$.

In agreement with this estimate is the shape of the radio spectrum from VLA and MERLIN observations, which indicates a low frequency turnover at around $1.5 \mathrm{GHz}$ (Chapman et al. 1990). Such a turnover in the spectrum could be caused by either free-free absorption or synchrotron self-absorption (SSA). If we assume SSA, the the size (460 mas) and peak flux density (38 mJy) of the diffuse emission would require an unrealisticly large magnetic field strength $B \sim 10^{10} \mathrm{G}$, and conditions very far from equipartition. In contrast, if we assume the spectrum turnover is due to free-free absorbing gas and adopt (from the spectrum) a turnover frequency of $1.5 \mathrm{GHz}$, the emission measure equals $7 \times 10^{6} \mathrm{~cm}^{-6} \mathrm{pc}$. This is consistent with the independent measurement made by assuming that the Eastern and Western sides of the $\mathrm{OH}$ maser ring are equally bright.

Given the above emission measure and the ring geometry, the difference in absorbing path lengths between the Western and Eastern side of the maser ring is $\sim 38$ pc. Assuming the gas is evenly distributed along the line of sight with a volume filling factor $f$, we derive a lower limit to the density of $\sim 4 \times 10^{2} \mathrm{f}^{-1 / 6} \mathrm{~cm}^{-3}$. Assuming spherical symmetry over a radius of $22 \mathrm{pc}$ the upper limit of the estimated ionised mass is then $0.5 \times 10^{6} f^{5 / 6} M_{\odot}$. This is less than the total dynamical mass estimated in Sect. 5.2.

Alternatively of course, it is possible that we observe the true distribution of supernovae, with the supernovae mainly on the near side. The Eastern part of the $\mathrm{OH}$ ring would then also be weaker because the FIR pumping could be less due to a greater distance from the supernovae.

\section{Summary and conclusions}

From the results presented in this paper we can conclude that the continuum source in III Zw 35 is broadly consistent with emission originating in a starburst. Both diffuse and compact continuum emission is detected. The former we argue comes from electrons accelerated in SNRs; the latter from individual very powerful RSNe or clusters of young SNRs. The radio emission, in particular the compact component, is concentrated to the Western side of the source; just outside the ring of $\mathrm{OH}$ maser emission. We suggest that if the star formation is concentrated in a disk with its near edge to the West, this bias is plausibly due to free-free absorption. If this is true, deeper higher frequency continuum observations should show more compact components on the Eastern side.

This starburst, seen in the continuum, can presumably provide the IR photons needed to pump the observed $\mathrm{OH}$ maser emission. In the standard model, the $\mathrm{OH}$ maser emission in ULIRGs have been thought to be due to low-gain amplification of a background radio source, via an extended molecular screen on scales 100-1000 pc pumped by such starbursts. Recently, VLBI observations of for example the compact maser emission observed in Arp 220, have put this model in doubt. Using highsensitivity telescopes of EVN and MERLIN, we have presented new observations of the $\mathrm{OH}$ maser emission in III Zw 35 which broadly support the standard model, albeit in a very compact region. This is the first clear detection of $\mathrm{OH}$ maser emission in a thick rotating ring, and is the most compact example detected. We argue that the difference between compact and diffuse maser emission in III Zw 35 is not due to physical differences for instance in pumping or in number densities. Instead we suggest that this effect could be due to a geometrical effect, arising in the symmetric ring. Our observations place the compact masers at the tangent points of this ring, which have the longest path length along the line of sight.

A ring geometry explains the coexistence of diffuse and compact maser components in III Zw 35. The situation in the ULIRG prototype Arp 220 slightly resembles the one in IIIZw 35; in Arp 220 a dual nature for the $1667 \mathrm{MHz}$ maser emission is also observed (Lonsdale et al. 1998). Moreover, VLBI experiments have resolved out one third of the single-dish flux. Plausibly, further high-sensitivity EVN+MERLIN observations of Arp 220 covering the missing baseline ranges might reveal a similar ring geometry as in III Zw 35 . On the other hand, the results on the $1665 \mathrm{MHz}$ emission in III Zw 35 differ from the results obtained in Arp 220, where Lonsdale et al. (1998) in their VLBI maps detected none of the single dish $1665 \mathrm{MHz}$ emission in either diffuse or compact components, and derived a lower limit of the $1667 / 1665 \mathrm{MHz}$ flux ratio of 100 . The non-detection was interpreted to mean that all of the $1665 \mathrm{MHz}$ emission must be very extended and resolved out. This is obviously not the case in III Zw 35 where the $1665 / 1667 \mathrm{MHz}$ line ratios measured in our images are comparable to the single dish ratios.

We note that the appearance of two spots connected by weaker arcs in our model for III Zw 35 will only occur for a torus with a certain range of inclinations. At large inclinations the near and the far side of the ring will overlap and the difference between the apparent brightness at the tangent points and along the arcs will be reduced. A similar effect will result when the inclination is small and the disk is close to face on since all lines of sight will then have similar path length. Speculating that all $\mathrm{OH}$ megamasers galaxies harbour nuclear $\mathrm{OH}$ rings, only the ones with optimum orientation will thus display both compact and diffuse maser emission (for instance Arp 220 and III Zw 35). Other orientations would produce smoother maser emission, which could perhaps be the case in Mrk 273 (Yates et al. 2000), in the Seyfert 1 classified Mrk 231 (H. Klöckner, private comm.), and in IC694 (Polatidis \& Aalto 2000).

In this paper we have shown that high resolution radio continuum and spectral line observations provide a unique means of studying in detail the structure and dynamics 
of ultra-compact starburst galaxies. Compared to any other wavebands there is much less obscuration. We hope to continue to study in detail the starburst in III Zw 35 using multi-frequency radio continuum and observations in other wavebands. III Zw 35 also shows $\mathrm{H}$ I absorption (Mirabel \& Sanders 1987). It will be interesting to map the $\mathrm{H}$ I absorption to see if it has a velocity gradient in the same sense as that of the $\mathrm{OH}$, which has been observed in for example Mrk 273 (Cole et al. 1999; Carilli \& Taylor 2000) and IC 694 (Polatidis \& Aalto 2000). We also hope to model in detail the maser emitting gas and provide a general model of gas conditions in this ultra-compact starburst.

Acknowledgements. The European VLBI Network is a joint facility of European and Chinese radio astronomy institutes funded by their national research councils. Y. M. P. wishes to acknowledge Chalmers University of Technology for financial support during this project, which constitutes a part of a Ph.D. project.

\section{References}

Carilli, C. L., \& Taylor, G. B. 2000, ApJ, 533, L13

Chapman, J. M., Staveley-Smith, L., Axon, D. J., et al. 1990, MNRAS, 244, 281

Cole, G. H. J., Pedlar, A., Holloway, A. J., \& Mundell, C. G. 1999, MNRAS, 3101033

Condon, J. J., \& Broderick, J. J. 1988, AJ, 96, 30

Condon, J. J., \& Yin, Q. F. 1990, ApJ, 357, 97

Condon, J. J. 1992, ARA\&A, 30, 575

Diamond, P. J., Lonsdale, C. J., \& Smith, H. E. 1999, ApJ, 511,178

Genzel, R., Lutz, D., Sturm, E., et al. 1998, ApJ, 498, 579
Helou, G., Soifer, B. T., \& Rowan-Robinson, M. 1985, ApJ, 298, L7

Lonsdale, C. J., Lonsdale, C. J., Diamond, P. J., \& Smith, H. J. 1998, ApJL, 493, L13

Meurer, G. R., Heckman, T. M., Leitherer, C., et al. 1995, AJ, 110,2665

Mirabel, I. F., \& Sanders, D. B. 1987, ApJ, 322, 688

Montgomery, A. S., \& Cohen, R. J. 1992, MNRAS, 254, 23

Panagia, N., Weiler, K. W., Montes, M. J., et al. 2001, to appear in Proceedings of the Volcano Workshop, Italy

Pedlar, A. 2000, in Proceedings of the 5th EVN Symp., ed. J. Conway, A. Polatidis, R. Booth, \& Y. Pihlström, Onsala Space Observatory, Chalmers University of Technology (Gothenburg, Sweden)

Polatidis, A., \& Aalto, S. 2000, in Proceedings of the 5th EVN Symp., ed. J. Conway, A. Polatidis, R. Booth., \& Y. Pihlström, Onsala Space Observatory, Chalmers University of Technology (Gothenburg, Sweden)

Randell, J., Field, K. N., Jones, K. N., Yates, J. A., \& Gray, M. D. 1995, A\&A, 300, 659

Sanders, D. B., Scoville, N. Z., \& Soifer, B. T. 1991, ApJ, 370 , 158

Smith, H. E., Lonsdale, C. J., \& Lonsdale, C. J. 1998, ApJ, 492, 137

Smith, H. E., Lonsdale, C. J., Lonsdale, C. J., \& Diamond, P. J. 1998, ApJ, 493, L17

Staveley-Smith, L., Cohen, R. J., Chapman, J. M., Pointon, L., \& Unger, S. W. 1987, MNRAS, 226, 689

Trotter, A. S., Moran, J. M., Greenhill, L. J., Zheng, X., \& Gwinn, C. R. 1997, ApJ, 485, L79

Veilleux, S., Kim, D.-C., Sanders, D. B., Mazzarella, J. M., \& Soifer, B. T. 1995, ApJS, 98, 171

Weiler, K. W., Panagia, N., \& Sramek, R. 1990, ApJ, 364, 611

Yates, J. A., Richards, A. M. S., Wright, M. M., et al. 2000, MNRAS, 317, 28 